\title{
Reprodução da exótica rã-touro Lithobates catesbeianus (Shaw, 1802) (Amphibia, Anura, Ranidae) em riachos de Mata Atlântica no estado de Minas Gerais, Brasil
}

\author{
Lilian Gomes Afonso ${ }^{1 *}$ \\ Rodolfo Carvalho ${ }^{1}$ \\ Fernando Morais dos Santos ${ }^{1}$ \\ Ana Carolina Ballesteros Coelho ${ }^{1}$ \\ André Lincoln Barroso de Magalhães ${ }^{1,2}$ \\ ${ }^{1}$ Centro Universitário UNA \\ Rua Guajajaras, 175, CEP 30180-100, Belo Horizonte - MG, Brasil \\ ${ }^{2} \mathrm{PPG}$ em Ecologia, Conservação e Manejo de Vida Silvestre \\ Universidade Federal de Minas Gerais, Belo Horizonte - MG, Brasil \\ *Autor para correspondência \\ lgafonso@gmail.com
}

Submetido em 15/11/2009

Aceito para publicação em 23/04/2010

\section{Resumo}

A reprodução da exótica rã-touro Lithobates catesbeianus foi estudada bimestralmente de janeiro a dezembro de 2005 e 2006 analisando-se girinos, imagos e vocalização de adultos em três riachos localizados no município de Vieiras, estado de Minas Gerais, Brasil. Os girinos foram classificados nas fases de desenvolvimento G1 (sem patas), G2 (com patas traseiras), G3 (com patas dianteiras e traseiras), I (imago) e calcularam-se suas frequências absoluta e relativa por bimestre e por riacho. Os resultados mostraram que nos bimestres mais quentes houve maior proporção de girinos G1, G2 e G3 nos riachos Santo Antônio e Chato e G1, G3 e I no riacho Gavião. Girinos nas fases G1 e G2 predominaram nos bimestres mais frios em todos os locais. Vocalizações de machos juntamente com maiores médias de temperatura da água e pluviometria foram registradas nos 3 riachos durante os bimestres mais quentes. Medidas devem ser adotadas para acabar com os animais introduzidos e evitar novas re-introduções na região, tais como: (1) cercar os recintos onde adultos se localizam; (2) colocar telas protetoras nos canos efluentes que liberam água dos tanques diretamente nos riachos, (3) promover esclarecimentos para as pessoas que trabalham nas pisciculturas ornamentais sobre a problemática das invasões biológicas.

Unitermos: Brasil, invasões biológicas, Lithobates catesbeianus, rã-touro, reprodução

\section{Abstract}

Reproduction of the exotic bullfrog Lithobates catesbeianus (Shaw, 1802) (Anura, Ranidae) in creeks of the Atlantic Rainforest of Minas Gerais, Brazil. Reproduction of the exotic bullfrog Lithobates catesbeianus was studied every two months from January 2005 to December 2006, analyzing tadpoles, juveniles, and adult vocalizations at three creeks located in the largest Brazilian center of ornamental fish breeding. Absolute and 
relative tadpole frequencies were measured bimonthly in three development stages: G1 (no limbs), G2 (hind limbs present), G3 (fore and hind limbs) and I (metamorphs). Results show that, during warmest months, a greater proportion of tadpoles in G1, G2, and G3 phases were found in Santo Antônio and Chato creeks, while G1, G3, and I phases were more common in Gavião creek. Tadpoles in G1 and G2 phases predominated in coldest months at all locations. Male calls and highest average water temperatures and rainfall were recorded at 3 streams during the hottest months. Management measures should be adopted, such as the extermination of larval and adult stages, and initiatives should be taken to prevent new re-introductions, such as: (1) installation of fences around tanks where adults are located, (2) placement of protective screens on the pipes that release waste water from tanks directly into streams, (3) capacity building and educational measures on the problems of biological invasions for staff working on ornamental fish farms.

Key words: biological invasions, Brazil, bullfrog, Lithobates catesbeianus, reproduction

\section{Introdução}

Existem organismos que não estão ocupando todos os seus habitats potenciais e, caso sejam transferidos para outros ambientes, podem sobreviver, reproduzir e se espalhar (Krebs, 1985). A introdução de espécies em novos habitats, seja deliberadamente ou acidental, provoca consequências devastadoras e devem ser evitadas (Begon et al., 1996).

A espécie Lithobates catesbeianus é nativa da América do Norte, do sul de Quebec no Canadá ao leste dos Estados Unidos. Ela foi introduzida em muitas regiões dos Estados Unidos e no México, Cuba, Jamaica, Porto Rico, Bermuda, Honduras, Guatemala, El Salvador, Panamá, Colômbia, Equador, Paraguai, Chile, Itália, França, Bélgica, Holanda, Reino Unido, Espanha, China, Taiwan e Japão (Frost, 2009). No Brasil, a espécie foi detectada exclusivamente nos biomas Mata Atlântica e Cerrado, nos estados do Piauí, Alagoas, Goiás, Minas Gerais, Espírito Santo, Rio de Janeiro, São Paulo, Paraná, Santa Catarina e Rio Grande do Sul (Instituto Horus, 2007; Giovanelli et al., 2008).

Este anuro, quando introduzido com sucesso, forma densas populações com alta capacidade adaptativa. Cada fêmea pode depositar até vinte mil ovos, desovando várias vezes ao ano (Vizotto, 1984). Os ovos e as larvas apresentam uma série de substâncias que fazem com que tenham um sabor desagradável para os predadores (Rodriguez e Linares, 2001). Esta espécie pode viver praticamente em qualquer ambiente, como rios, riachos, lagoas, mesmo com elevados níveis de poluição, além de ter grande longevidade, entre sete e nove anos, o que dá grandes vantagens adaptativas em qualquer meio (Rodriguez e Linares, 2001).
Sua dieta é composta essencialmente de todos os tipos de insetos, mas incluem-se também pequenos mamíferos como ratos e morcegos, aves, répteis e outras espécies de anfíbios (Rodriguez e Linares, 2001). Quando o alimento é escasso, pratica canibalismo. Os girinos são herbívoros vorazes e geralmente desequilibram os delicados ambientes dulceaquícolas (Instituto Horus, 2007).

Estudos relativos a aspectos da reprodução de $L$. catesbeianus introduzido em corpos d'água da Mata Atlântica no Brasil são praticamente inexistentes, estando restritos apenas aos estados do Paraná, Santa Catarina e Rio Grande do Sul (Conte e Rossa-Feres, 2006; Kaefer et al., 2007; Alves et al., 2008). Além de contribuir para minimizar essa lacuna e seguindo uma das metas específicas do programa Biota Minas para organismos aquáticos que é ampliar o conhecimento sobre as invasões biológicas e seus mecanismos de controle no estado de Minas Gerais (Drummond et al., 2009), os objetivos desta pesquisa são: 1) estudar a reprodução (frequência das fases de desenvolvimento de girinos e vocalização sazonal de machos adultos relacionado a fatores abióticos) da exótica rã-touro Lithobates catesbeianus em riachos de Mata Atlântica pertencentes à bacia do rio Paraíba do Sul, estado de Minas Gerais, sudeste do Brasil e 2) sugerir medidas para se evitar futuras re-introduções na região.

\section{Materiais e Métodos}

As coletas de girinos e imagos foram realizadas bimestralmente de janeiro de 2005 a dezembro de 2006 em três riachos permanentes: riacho Santo Antônio 
(20 $\left.56^{\prime} \mathrm{S} ; 42^{\circ} 17^{\prime} \mathrm{W}\right)$, riacho Chato $\left(20^{\circ} 58^{\prime} \mathrm{S} ; 4^{\circ} 17^{\prime} \mathrm{W}\right)$ e riacho Gavião (20 $\left.0^{\circ} 9^{\prime} \mathrm{S} ; 42^{\circ} 17^{\prime} \mathrm{W}\right)$. Todos os riachos pertencem à sub-bacia do rio Glória, município de Vieiras, estado de Minas Gerais (Figura 1), sendo que o riacho Chato dista, aproximadamente, $1,5 \mathrm{~km}$ do riacho Gavião e 6km do riacho Santo Antônio.

As capturas foram realizadas com peneiras $(90 \mathrm{~cm}$ de comprimento, $75 \mathrm{~cm}$ de altura, malha de $0,5 \mathrm{~cm}$ ). Após as coletas, todos os exemplares foram fixados em formol $10 \%$ e posteriormente preservados em álcool $70 \%$. Três exemplares testemunho de cada fase de desenvolvimento foram coletados e depositados na coleção de Herpetologia do Museu de Ciências Naturais da Pontifícia Universidade Católica de Minas Gerais.

Seguindo terminologia proposta por Lima e Agostinho (1992), os girinos foram classificados nas fases de desenvolvimento G1 (sem patas), G2 (com patas traseiras), G3 (com patas dianteiras e traseiras) e I (imago). As frequências absoluta e relativa de cada fase de desenvolvimento foram em seguida calculadas por bimestre e por riacho.
Durante a captura de girinos e imagos eram registradas as vocalizações de machos adultos nos três riachos estudados.

A temperatura da água foi medida durante três dias consecutivos, por bimestre, com termômetro de filamento de mercúrio com escala de 0 a $50^{\circ} \mathrm{C}$ em um ponto fixo de cada riacho analisado no período de $8 \mathrm{~h}$ às 10h, a média foi obtida somando os valores de cada mês e dividindo por dois. A média bimestral de precipitação pluviométrica (milímetros) das regiões onde estão inseridos os riachos foi cedida pela plataforma de coleta de dados de Vieiras fornecidas pelo INPE (2009).

Foi utilizado o teste de Shapiro-Wilk para verificar a normalidade dos dados. Como estes apresentaram distribuição normal foi utilizado o índice de correlação de Pearson para verificar se a vocalização de machos adultos esteve relacionada com a temperatura média da água dos riachos e a pluviometria das regiões. Esta análise foi realizada pelo programa Past versão 1.85 (Hammer et al., 2001).

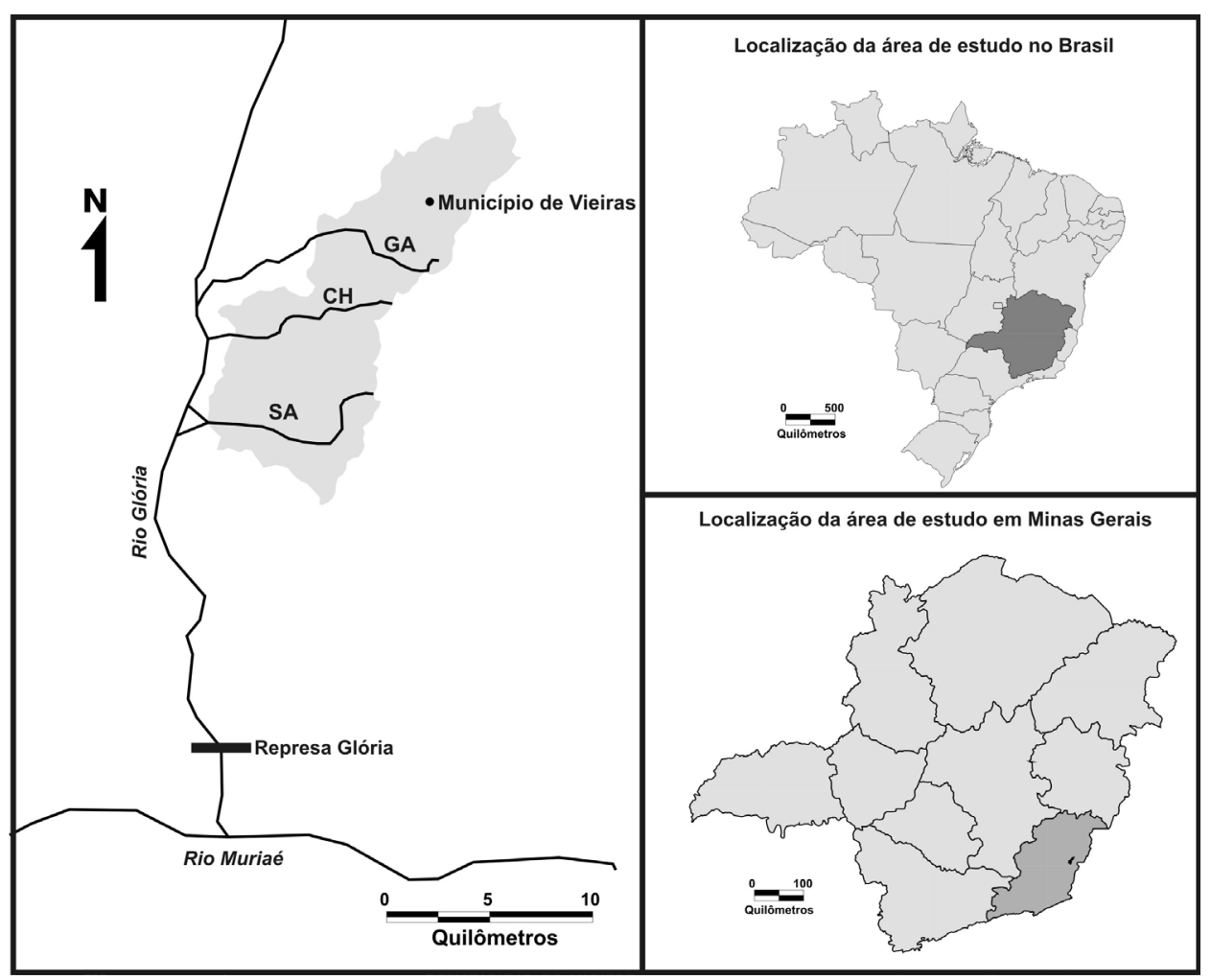

FIGURA 1: Mapa mostrando os três locais de estudo no estado de Minas Gerais, Brasil: riacho Santo Antônio (SA), riacho Chato (CH) e riacho Gavião (GA). 


\section{Resultados e Discussão}

Ao todo, 67 girinos foram coletados no Riacho Santo Antônio, 38 girinos no Riacho Chato e 55 girinos e seis imagos no Riacho Gavião.

Não foram capturados adultos nos riachos, mas as vocalizações de machos foram registradas durante 0 período de estudo (Tabela 1).

A análise da distribuição bimestral da frequência das fases de desenvolvimento mostrou que nos bimestres mais quentes (novembro/dezembro, janeiro/fevereiro, março/abril), houve maior proporção de girinos nas fases G1, G2, G3 nos riachos Santo Antônio e Chato, e girinos nas fases G1, G3 e imagos no riacho Gavião. Girinos nas fases $\mathrm{G} 1$ e $\mathrm{G} 2$ predominaram nos bimestres mais frios (maio/junho, julho/agosto, setembro/outubro) em todos os locais (Figura 2). Vocalizações de machos adultos, juntamente com as maiores médias bimestrais de temperatura da água e pluviometria, foram registradas nos três riachos durante os bimestres mais quentes (novembro/dezembro, janeiro/fevereiro, março/abril) de 2005 e 2006 (Tabela 1).

Houve correlação significativa entre a temperatura da água dos riachos, pluviometria das regiões e a vocalização de machos adultos nos três ambientes analisados (Tabela 2).

A região onde girinos e imagos de L. catesbeianus foram registrados compreende 12 municípios e tem cerca de 250 produtores que criam 60 diferentes espécies de peixes ornamentais em mais de 3.000 tanques de terra e concreto (Rasguido e Albanez, 2000; Vidal Júnior e Costa, 2000). Há também, associado aos tanques de peixes ornamentais, o cultivo de plantas (Elodea spp., Valisneria spp., Azolla cf. microphylla, Eichornia crassipes, Salvinia auriculata, Pistia stratiotes), crustáceos (Procambarus clarkii, Uca rapax, Macrobrachium acanthurus), moluscos (Physa sp., Melanoides tuberculata, Pomacea bridgesii) e rãs como L. catesbeianus e Xenopus leavis, sendo estes anfíbios vendidos como animais ornamentais para as lojas de

TABELA 1: Registro de vocalização de machos adultos de Lithobates catesbeianus, média bimestral da temperatura da água e pluviometria dos riachos Santo Antônio, Chato e Gavião no período de janeiro de 2005 a dezembro de 2006, município de Vieiras, MG.

\begin{tabular}{|c|c|c|c|c|c|c|}
\hline Locais & $\mathbf{J} / \mathbf{F}$ & $\mathbf{M} / \mathbf{A}$ & $\mathbf{M} / \mathbf{J}$ & $\mathbf{J} / \mathbf{A}$ & $\mathrm{S} / \mathrm{O}$ & N/D \\
\hline \multirow{3}{*}{ Santo Antônio (2005) } & $\operatorname{sim}$ & $\operatorname{sim}$ & não & não & não & $\operatorname{sim}$ \\
\hline & $28^{\circ} \mathrm{C}$ & $28^{\circ} \mathrm{C}$ & $21^{\circ} \mathrm{C}$ & $20^{\circ} \mathrm{C}$ & $23^{\circ} \mathrm{C}$ & $31^{\circ} \mathrm{C}$ \\
\hline & $\begin{array}{c}195,33 \mathrm{~mm} \\
\operatorname{sim}\end{array}$ & $\begin{array}{l}150,35 \mathrm{~mm} \\
\operatorname{sim}\end{array}$ & $\begin{array}{c}16,33 \mathrm{~mm} \\
\text { não }\end{array}$ & $\begin{array}{c}\text { 5,33mm } \\
\text { não }\end{array}$ & $\begin{array}{c}\text { 20,63mm } \\
\text { não }\end{array}$ & $\begin{array}{c}210,35 \mathrm{~mm} \\
\operatorname{sim}\end{array}$ \\
\hline \multirow[t]{2}{*}{ Chato (2006) } & $29^{\circ} \mathrm{C}$ & $26^{\circ} \mathrm{C}$ & $21^{\circ} \mathrm{C}$ & $22^{\circ} \mathrm{C}$ & $22^{\circ} \mathrm{C}$ & $28^{\circ} \mathrm{C}$ \\
\hline & $\begin{array}{l}193,83 \mathrm{~mm} \\
\quad \operatorname{sim}\end{array}$ & $\begin{array}{l}113,54 \mathrm{~mm} \\
\quad \operatorname{sim}\end{array}$ & $\begin{array}{c}\text { 11,04mm } \\
\text { não }\end{array}$ & $\begin{array}{c}\text { 12,04mm } \\
\text { não }\end{array}$ & $\begin{array}{c}\text { 74,54mm } \\
\text { não }\end{array}$ & $\begin{array}{l}126,00 \mathrm{~mm} \\
\quad \operatorname{sim}\end{array}$ \\
\hline \multirow[t]{2}{*}{ Gavião (2006) } & $30^{\circ} \mathrm{C}$ & $26^{\circ} \mathrm{C}$ & $23^{\circ} \mathrm{C}$ & $20^{\circ} \mathrm{C}$ & $22^{\circ} \mathrm{C}$ & $29^{\circ} \mathrm{C}$ \\
\hline & $193,8 \mathrm{~mm}$ & $113,54 \mathrm{~mm}$ & $11,04 \mathrm{~mm}$ & $12,04 \mathrm{~mm}$ & $74,54 \mathrm{~mm}$ & $126,00 \mathrm{~mm}$ \\
\hline
\end{tabular}

TABELA 2: Resultados do coeficiente de correlação de Pearson entre vocalização de machos adultos de Lithobates catesbeianus e variáveis ambientais nos riachos Santo Antônio, Chato e Gavião de janeiro de 2005 a dezembro de 2006.

\begin{tabular}{|c|c|c|c|c|}
\hline Riacho & $\begin{array}{c}\text { Indivíduos em } \\
\text { vocalização }\end{array}$ & Variáveis & Pearson (r) & Significância (p) \\
\hline \multirow{2}{*}{ Santo Antônio } & \multirow{2}{*}{$\mathrm{n}=7$} & \multirow{2}{*}{ temperatura da água pluviometria } & 0,874 & $0,022 *$ \\
\hline & & & 0,885 & $0,019 *$ \\
\hline \multirow{2}{*}{ Chato } & \multirow{2}{*}{$\mathrm{n}=10$} & \multirow{2}{*}{ temperatura da água pluviometria } & 0,977 & $0,000 *$ \\
\hline & & & 0,922 & $0,008 *$ \\
\hline \multirow{2}{*}{ Gavião } & \multirow{2}{*}{$\mathrm{n}=12$} & \multirow{2}{*}{ temperatura da água pluviometria } & 0,889 & $0,017 *$ \\
\hline & & & 0,920 & $0,009 *$ \\
\hline
\end{tabular}

* Significativo $(\mathrm{p}<0,05)$. 
aquários da região metropolitana de Belo Horizonte, Contagem e Betim (Magalhães, observação pessoal). Shiau et al. (2006) também confirmaram a utilização de $L$. catesbeianus para fins ornamentais em Taiwan, Ásia. De acordo com esses autores, a rã-touro é a terceira espécie de anfíbio mais frequente em lojas desse país, perdendo somente para Ceratophrys cranwelli e Ceratophrys sp. (híbrido).

Para $40 \%$ dos produtores brasileiros, a atividade de cultivo de plantas e animais ornamentais é a maior fonte de renda, o que torna esta região, o mais importante pólo da aquicultura ornamental na América do Sul desde o final da década de 70 (Vidal Júnior e Costa, 2000), e a principal fonte de introdução de plantas, invertebrados, peixes e anfíbios exóticos no continente (Magalhães, 2007; Magalhães e Carvalho, 2007). A presença de girinos nos riachos Santo Antônio e Chato e girinos e imagos no riacho Gavião foi devida, provavelmente, à fuga de adultos dos tanques de peixes ornamentais os quais posteriormente se adaptaram nos ambientes em questão. A mesma característica de introdução foi encontrada por Dixo e Verdade (2006) no estado de São Paulo.

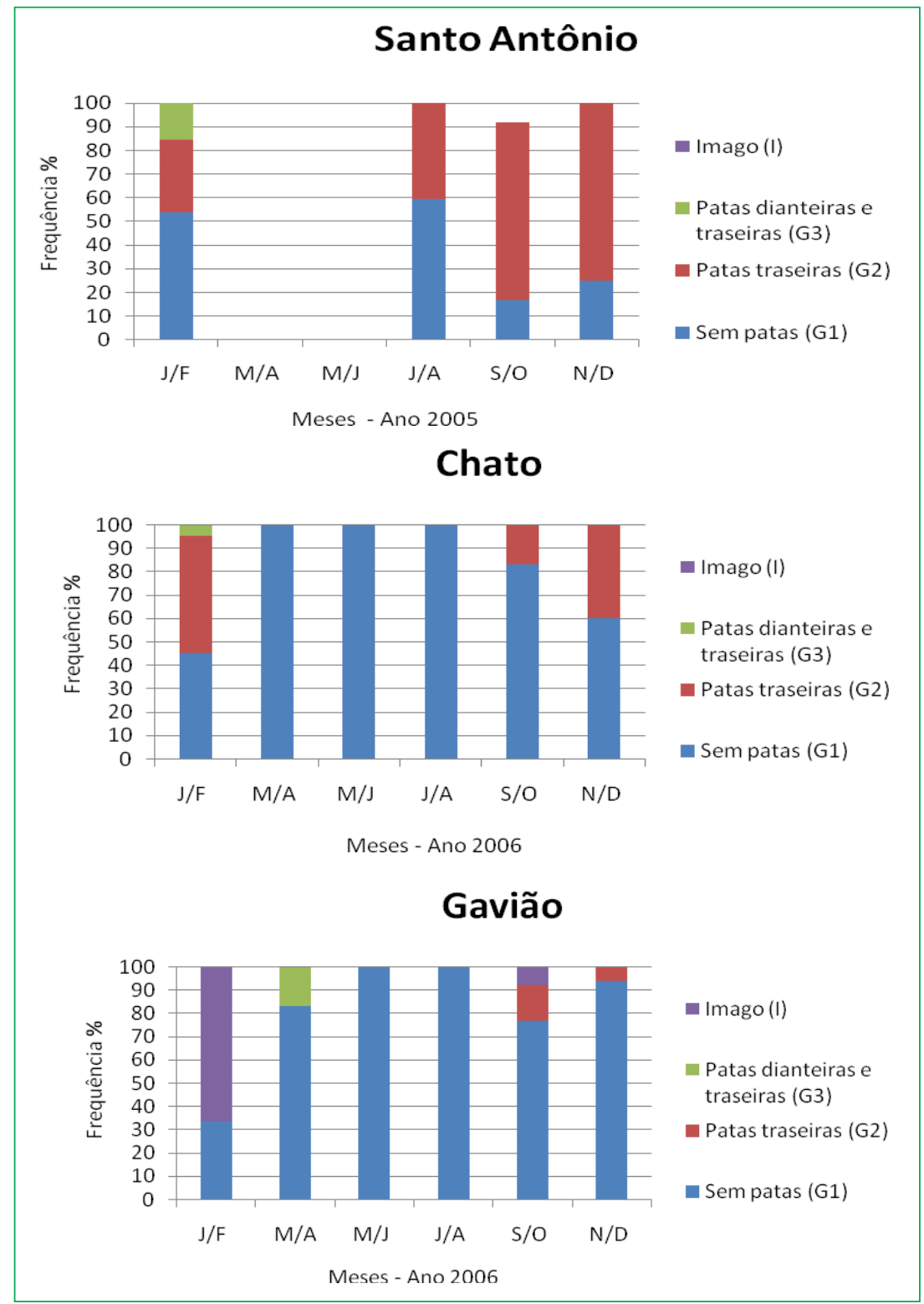

FIGURA 2: Frequência relativa bimestral das fases de desenvolvimento de Lithobates catesbeianus nos três riachos nos períodos de janeiro de 2005 a dezembro de 2006. 
Nossos resultados, associados à presença de vocalizações de machos adultos, médias bimestrais de temperatura da água e pluviometria mostraram que nos riachos Santo Antônio, Chato e Gavião, L. catesbeianus reproduz na estação quente (novembro/dezembro, janeiro/ fevereiro, março/abril), cessando o processo na estação fria (maio/junho, julho/agosto, setembro/outubro). Resultados semelhantes foram encontrados por Conte e Rossa-Feres (2006) e Kaefer et al. (2007), os quais relataram que L. catesbeianus introduzido no Paraná e Rio Grande do Sul, interrompiam sua atividade reprodutiva devido às baixas temperaturas de outono e inverno nestes estados. Os girinos coletados na fase G1 e G2 na época mais fria (provenientes de desovas no período quente) estavam com seu processo de metamorfose parado devido provavelmente à baixa temperatura da água dos três riachos, somente aumentando de tamanho e peso, o que pode gerar metamorfos e adultos maiores, fenômeno denominado overwintering (Pace, 1974). Santos et al. (2008), ao estudar o crescimento de girinos de rã-touro, verificaram que os animais mantidos em temperatura mais elevada $\left(30^{\circ} \mathrm{C}\right)$ apresentaram maior ganho de peso e menor tempo para concluírem a metamorfose.

Segundo Hilsdorf e Petrere Jr. (2002), uma das principais ameaças para a bacia do Rio Paraíba do Sul é a presença de espécies não-nativas. Desta forma, registros de espécies exóticas são necessários para se criar uma base de dados com o objetivo de preservar a fauna nativa de água doce desta bacia. Além disso, estudos relacionados à alimentação e densidade relativa de adultos, girinos e imagos de L. catesbeianus são necessários, a fim de se avaliar os impactos negativos sobre as espécies nativas da região, pois sua presença pode influenciar populações de anuros nativos devido à predação, à competição por recursos alimentares e à facilidade de reprodução (Alves et al., 2008). Outro risco a se considerar é que as rãs-touro podem atuar como vetores de microorganismos patogênicos, especialmente Batrachochytrium dendrobatidis, fungo responsável pela quitridiomicose, doença emergente sendo uma das principais causas de declínio e mortalidade de anfíbios no planeta (Daszak et al., 2003).

Os achados do presente trabalho são um alerta, não somente pelo risco ecológico da invasão de $L$. catesbeianus em um dos mais importantes hotspots de conservação do mundo, a Mata Atlântica, mas também pelos riscos sanitários implicados. No Brasil, a liberação de organismos exóticos em ambientes naturais é considerada crime ambiental (artigo 31 da Lei 9.605 de 1998) e a aquicultura ornamental no país deve ser conduzida em conformidade com regulamentações específicas (Magalhães et al., 2002), além do cumprimento efetivo da Lei Ambiental número 6.938 de 1991 a qual reje o princípio do "poluidor pagador". Algumas medidas corretivas devem ser feitas para acabar com animais introduzidos como exterminar fases larvais/ adultas nos ambientes e evitar novas re-introduções de rãs-touro na região, tais como: (1) cercar corretamente os tanques de piscicultura ornamental onde os adultos se localizam, (2) colocar telas protetoras nos canos efluentes que liberam água dos tanques diretamente nos riachos e, (3) promover esclarecimentos para as pessoas que trabalham diretamente nas pisciculturas ornamentais sobre as consequências da soltura de espécies exóticas.

Caso estas medidas não sejam adotadas, as introduções e provável adaptação da rã-touro, uma das cem piores espécies de animais exóticos do planeta (Lowe et al., 2000), continuarão na região.

\section{Agradecimentos}

Aos piscicultores ornamentais por permitirem nossa pesquisa em suas propriedades particulares. A professora Milena Wachlevski Machado (Fundação Educacional de Divinópolis/Universidade do Estado de Minas Gerais) pelas valiosas sugestões ao manuscrito.

\section{Referências}

Alves, F. C.; Branco, A.; Lucas, E. M.; Fortes, V. B. 2008. Ocorrência da espécie exótica Lithobates catesbeianus (rã-touro) em ambientes naturais nos municípios de Chapecó e Guatambu, Santa Catarina, Brasil. Acta Ambiental Catarinense, 5: 35-42.

Begon, M.; Harper, J. L.; Townsend, C. R. 1996. Ecology: Individuals, Populations and Communities. $3^{\text {rd }}$ edition. Blackwell Science, Oxford, UK, 1068pp.

Conte, C. E.; Rossa-Feres, D. C. 2006. Diversidade e ocorrência temporal da anurofauna (Amphibia, Anura) em São José dos Pinhais, Paraná, Brasil. Revista Brasileira de Zoologia, 23: 162175. 
Daszak, P.; Cunningham, A.; Hyatt, A. D. 2003. Infectious disease and amphibian population declines. Divesity and Distributions, 9: $141-150$.

Dixo, M.; Verdade, V. K. 2006. Herpetofauna de serrapilheira da Reserva Florestal de Morro Grande, Cotia (SP). Biota Neotropica, 6: 1-20.

Drummond, G. M.; Martins, C. S.; Greco, M. B. 2009. Biota Minas: Diagnóstico do conhecimento sobre a biodiversidade no estado de Minas Gerais - Subsídio ao programa BIOTA MINAS. Fundação Biodiversitas, Belo Horizonte, Brasil, 624pp.

Frost, D. R. 2009. Amphibian species of the world: An online reference. Disponível em <http://research.amnh.org/herpetology/ amphibia/index.html>. Acesso em 30 de setembro de 2009.

Giovanelli, J. G. R.; Haddad, C. F. B.; Alexandrino, J. 2008. Predicting the potential distribution of the alien invasive American bullfrog (Lithobates catesbeianus) in Brazil. Biological Invasions, 10: $585-590$.

Hammer, Ø.; Harper,D.A. T.; Ryan,P.D. 2001.Past:Palaeontological Statistic Software and data analysis. Palaeontologia Electronica, 4: $1-9$.

Hilsdorf, A. W. S.; Petrere Jr., M. 2002. Conservação de peixes na bacia do rio Paraíba do Sul. Ciência Hoje, 30: 62-65.

INPE - Instituto Nacional de Pesquisas Espaciais. 2009. Dados observacionais. Disponível em $<$ http://www.cptec.inpe.br/dados observados/>. Acesso em 01 de maio de 2009.

Instituto Horus. 2007. Base de dados de espécies exóticas invasoras no Brasil. Disponível em <http://www.institutohorus. org.br/pr_trabalhos.htm>. Acesso em 23 de abril de 2008.

Kaefer, I. L.; Boelter, R. A.; Cechin, S. Z. 2007. Reproductive biology of invasive bullfrog Lithobates catesbeianus in southern Brazil. Annales Zoologici Fennici, 44: 435-444.

Krebs, C. J. 1985. Ecology: The experimental analysis of distribution and abundance. $3^{\text {rd }}$ Harper Collins, New York, USA, 800pp.

Lima, S. L.; Agostinho, C. A. 1992. A tecnologia de criação de rãs. Editora Imprensa Universitária/UFV, Viçosa, Brasil, 168pp.
Lowe, S.; Browne, M.; Boudjelas, S.; De Poorter, M. 2000. 100 of the world's worst invasive alien species - A selection from the global invasive species database. School of Geography and Environmental Sciences, University of Auckland, Auckland, New Zealand, 12pp.

Magalhães, A. L. B. 2007. Pólo de piscicultura ornamental de Muriaé, Estado de Minas Gerais: Maior fonte dispersora de espécies exóticas do Brasil. Boletim da Sociedade Brasileira de Ictiologia, 86: 5-6.

Magalhães, A. L. B.; Amaral, I. B.; Ratton, T. F.; Brito, M. F. G. 2002. Ornamental exotic fishes in the Glória reservoir and Boa Vista Stream, Paraíba do Sul river basin, state of Minas Gerais, southeastern Brazil. Comunicações do Museu de Ciências e Tecnologia, 15: 265-278.

Magalhães, A. L. B.; Carvalho, P. A. 2007. Ocorrência de peixes ornamentais exóticos em riachos nos Estados de Minas Gerais e Rio de Janeiro, Brasil. Natureza \& Conservação, 5: 54-59.

Pace, A. E. 1974. Systematics and biological studies of the Leopard frogs (Rana pipiens complex) of the United States. Miscellaneous Publications, Museum of Zoology, 148:1-152.

Rasguido, J. E. A.; Albanez, J. R. 2000. Piscicultura em Minas Gerais. Informativo Agropecuário, 21: 32-37.

Rodriguez, J. A.; Linares, M. J. 2001. Rana toro e sapo marino: la amenaza que viene, los controles aduaneiros que se realizan em Canárias son insuficientes. Revista de La Conserjería de Política Territorial y Médio Ambiente, 21: 12-14.

Santos, A. J. G.; Melo, C. L.; Macêdo, P. B. 2008. Efeito do fotoperíodo e temperatura no crescimento de girinos da rã-touro gigante. Revista Brasileira de Engenharia de Pesca, 3: 92-99.

Shiau, T. W.; Hou, P. C.; Wu, S. H.; Tu, M. C. 2006. A survey on alien pet reptiles in Taiwan. Taiwana, 51: 71-80.

Vidal Júnior, M. V.; Costa, S. M. 2000. A produção de peixes ornamentais em Minas Gerais. Informativo Agropecuário, 21: 44-47.

Vizotto, L. D. 1984. Ranicultura. Ciência e Cultura, 36: 42 - 45. 